\title{
Particle Filtering for On-Line Estimation of Overflow Losses in a Hopper Dredger
}

\author{
Robert Babuška, Zsofia Lendek, Jelmer Braaksma and Cees de Keizer
}

\begin{abstract}
A particle filter is applied to the estimation of overflow losses in a hopper dredger. The filter estimates online the overflow mixture density and flow-rate, based on the measurements of the total hopper volume, mass, incoming mixture density and flow-rate. These data are readily available on board of every modern hopper dredger. The main advantage of the proposed approach is that the particle filter uses straightforward nonlinear mass balance equations and does not rely on complex sedimentation models with uncertain parameters. The performance was evaluated in simulations as well as with real measurements and the results are encouraging. The filter can be used to improve parameter estimation in complex mechanistic models of the hopper sedimentation process and to facilitate decision making on board of the hopper dredger.
\end{abstract}

\section{INTRODUCTION}

The optimization of dredging operations is of vital importance for future improvements in efficiency, accuracy and labor saving. This article addresses the estimation of overflow losses, which is an essential step toward the optimization of the separation process in the hopper. While modern hopper dredgers are equipped with advanced dynamic positioning and tracking systems, no on-board decision-support systems are yet available to optimize the dredging performance under the given operating conditions (type of soil, dredging depth, water current, etc.). Settings of the manipulated variables must constantly be adjusted by two operators: the ship navigator and the dredge process operator. Consequently, the performance and efficiency of the entire process heavily depend on their insight and experience.

IHC Systems, a company specialized in the development and manufacturing of automation systems for dredgers, currently cooperates with the Delft Center for Systems and Control on the development of an adaptive decision-support system for hopper dredgers to advise the operators on the most suitable control strategy, given a specified performance goal. This can be, for instance, the minimization of the integral dredging costs per $\mathrm{m}^{3}$ of sand or the maximization of the production per time unit. To this end, a control-oriented dynamic model of the hopper dredger has been developed and calibrated by using recorded process data. Based on this model, a suitable control strategy can be derived, for instance, by using model-predictive control. As only some of the state variables are measured by sensors, the use of online state estimation techniques is essential for an on-board application of this system.

R. Babuška, Zs. Lendek and J. Braaksma are with Delft Center for Systems and Control, Delft University of Technology, Mekelweg 2, 2628 CD Delft, The Netherlands, r.babuska@dcsc.tudelft.nl.

C. de Keizer is with IHC Systems B.V. P.O. Box 41, 3360 AA Sliedrecht, The Netherlands, cdkeizer@ihcsystems.com.
Prior to stating the estimation problem, the principle of the dredging process is briefly explained. The dredger uses a drag head to excavate soil from the sea bottom. A mixture of soil and water is transported through a pipe to the hopper, which is a large storage tank inside the ship (see Fig. 1).

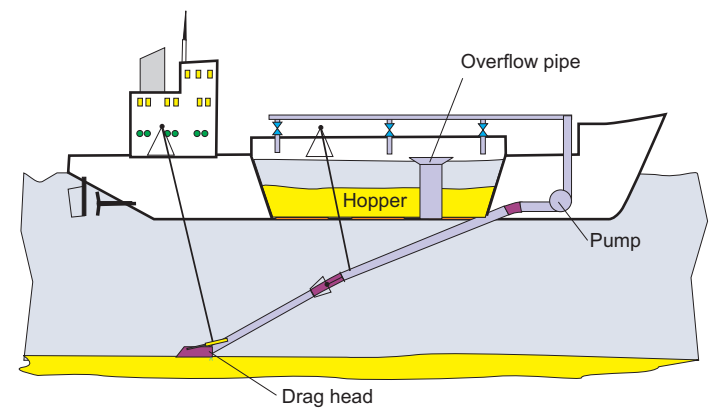

Fig. 1. Schematic drawing of a hopper dredger.

The soil gradually settles at the bottom of the hopper, while excessive water (in fact low-density mixture) is discharged through an overflow pipe whose level can be adjusted (see Fig. 2). As the height of the settled sand layer rises, so does the concentration of the overflow mixture and eventually the losses become so high that it is no longer economical to continue dredging. The ship then sails back to deliver the load. After the sand is discharged, the ship sails again to the dredging location and the whole cycle repeats.

The efficiency of the sedimentation process heavily depends on the type of soil and is influenced by the flowrate and density of the incoming mixture and the manner the overflow pipe is controlled. An important factor in the optimization of the dredging performance is the minimization of the overflow losses. This has been recognized long ago, and in the literature, a number of sedimentation models have been proposed [1], [2], [3], [4]. These models, however, cannot be used as a basis for control or optimization of the dredging process. The reason is that they are based on detailed (often PDE) modeling of the physical phenomena and contain too many uncertain parameters. Therefore, we propose to use simplified models, along with advanced signal processing and estimation techniques.

Information on the amount of overflow losses is essential both for decision support and automatic control. Unfortunately, these losses cannot be reliably measured, due to the presence of air in the overflow pipe. However, as shown in this paper, they can be estimated by using mathematical models and the available on-line measurements. Two methods have been investigated: 
1) By using a model of the density profile in the mixture above the sand bed, the overflow density can be computed. The overflow rate can be computed based on a hydraulic model. However, these models are highly uncertain and contain parameters that cannot be directly measured or estimated from on-line data.

2) Using volume and mass balance equations, the losses can be estimated by means of a nonlinear state observer (particle filter). As the balance equations do not contain any uncertain parameters, one only needs to tune the observer parameters.

Both methods are presented, but as latter one proved to be much more accurate and easier to use, detailed results are given for this method only. Section II presents the dynamic sedimentation model. In Section III, the estimation problem is given and Section IV reviews the particle filtering methodology. Section V gives the results and Section VI concludes the paper.

\section{Dynamic Sedimentation Model}

A dynamic state-space model of the sedimentation process has been developed, based on [1], [3], [4]. In the sequel, all variables are functions of time and the time argument is left out for notational simplicity. Derivatives with respect to time are denoted by a dot. Refer to the Appendix for a complete list of symbols.

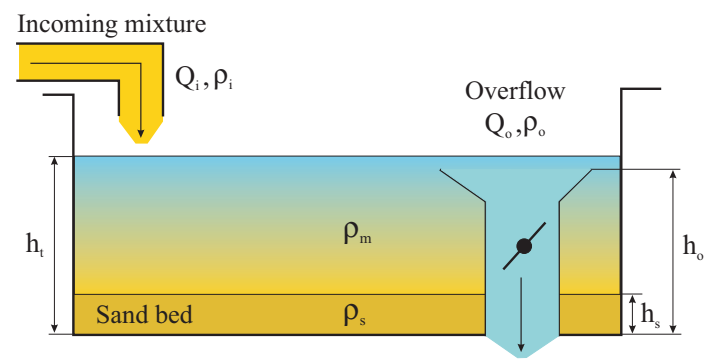

Fig. 2. The sedimentation process in the hopper.

A schematic diagram of the hopper is given in Fig. 2. The model has three states variables: the total mass in the hopper $m_{t}$, the total volume $V_{t}$ of the mixture in the hopper and the mass of the sand bed $m_{s}$. While the first two states can be derived from on-line measurements (the ship draught and the total level $h_{t}$, respectively), the mass of the sand bed is not measurable. The flow-rate $Q_{i}$ of the incoming mixture and the overflow height $h_{o}$ are the manipulated inputs and the incoming mixture density $\rho_{i}$ is in this context regarded as a measured disturbance. The volume and mass balance equations are given by:

$$
\begin{aligned}
\dot{V}_{t} & =Q_{i}-Q_{o} \\
\dot{m}_{t} & =Q_{i} \rho_{i}-Q_{o} \rho_{o} .
\end{aligned}
$$

The rate of material sedimentation is as a function the settling velocity $\left(f_{s}\right)$ and the scouring (erosion) effects $\left(f_{e}\right)$

$$
\dot{m}_{s}=f_{s}\left(\rho_{m}\right) f_{e}\left(Q_{o}, h_{m}\right) \text {. }
$$

The overflow rate $Q_{o}$, the density $\rho_{o}$ and the functions in (3) are modeled by using static relationships as detailed below.

\section{A. Overflow Rate}

If the outgoing mixture freely flows through the overflow pipe, the flow-rate $Q_{o}$ is given by [5]:

$$
Q_{o}=k_{o} \max \left(h_{t}-h_{o}, 0\right)^{\frac{3}{2}}
$$

where $k_{o}$ is an uncertain parameter depending on the overflow pipe shape and circumference. However, if the overflow pipe is full (e.g., because a valve inside the pipe is engaged), the following model must be used:

$$
Q_{o}=k_{o}^{\prime} \sqrt{2 g \max \left(h_{t}-h_{o}, 0\right)} .
$$

Clearly, there is a some uncertainty in the modeling of the overflow rate. Moreover, due to the model's switching nature, it is not straightforward to estimate its parameters.

\section{B. Overflow Density}

The density profile in the mixture above the sand bed can be approximated as a decreasing function of the height above the sand. The exact form of this function is highly uncertain and time varying. In this paper, we use the following linear approximation:

$$
\rho_{o}=\max \left(\rho_{s}-k_{\rho}\left(h_{o}-h_{s}\right), \rho_{w}\right) .
$$

The slope $k_{\rho}$ must be determined at every time instant such that the average mixture density $\rho_{m}$, computed from the mass-balance relations, equals to the average of the density profile:

$$
\rho_{m}=\frac{1}{h_{m}} \int_{h_{s}}^{h_{t}} \max \left(\rho_{s}-k_{\rho}\left(h-h_{s}\right), \rho_{w}\right) \mathrm{d} h
$$

with $h_{m}=h_{t}-h_{s}$. Solving this constraint for the linear model (6) yields the following equation for the slope:

$$
k_{\rho}= \begin{cases}\frac{2\left(\rho_{s}-\rho_{m}\right)}{h_{m}} & \text { for } \rho_{m}>\frac{1}{2}\left(\rho_{w}+\rho_{s}\right) \\ \frac{\left(\rho_{s}-\rho_{w}\right)^{2}}{2 h_{m}\left(\rho_{m}-\rho_{w}\right)} & \text { otherwise }\end{cases}
$$

where the average mixture density is given by:

$$
\rho_{m}=\frac{m_{t}-m_{s}}{V_{t}-\frac{m_{s}}{\rho_{s}}}=\frac{\rho_{s}\left(m_{t}-m_{s}\right)}{V_{t} \rho_{s}-m_{s}} .
$$

Validation based on measured data has shown that this model is not very accurate, but it suffices for the tuning and first evaluation of the particle filter.

\section{Settling and scouring functions}

The settling and scouring functions used in the sedimentation rate equation (3) are taken from [6], [7], [4]. The settling function $f_{s}$ describes how the rate of sedimentation depends on the undisturbed settling velocity $v_{s}$ and the mixture density:

$$
f_{s}\left(\rho_{m}\right)=A \rho_{s} v_{s} \frac{\rho_{m}-\rho_{w}}{\rho_{s}-\rho_{m}}\left(\frac{\rho_{q}-\rho_{m}}{\rho_{q}-\rho_{w}}\right)^{\beta} .
$$

The scouring function describes the effect of erosion on the sand bed due to the flow in the mixture (which is considered to be equal to the overflow rate in steady state):

$$
f_{e}\left(Q_{o}, h_{m}\right)=\max \left(1-\frac{Q_{o}^{2}}{k_{c} h_{m}^{2}}, 0\right) .
$$


The parameters of the entire model have been determined by fitting the outputs of the simulation model to real data from a ship, by using non-linear least-squares optimization.

\section{The Estimation Problem}

The volume and mass balance equations were discretized by using the Euler method:

$$
\begin{aligned}
V_{t, k+1} & =V_{t, k}+T_{s}\left(Q_{i, k}-Q_{o, k}\right) \\
m_{t, k+1} & =m_{t, k}+T_{s}\left(Q_{i, k} \rho_{i, k}-Q_{o, k} \rho_{o, k}\right)
\end{aligned}
$$

where the sampling period is $T_{s}=5 \mathrm{~s}$, which is also the sampling period of the on-board data acquisition system. The state equations are augmented with a random-walk model for $Q_{o}$ and $\rho_{o}$ :

$$
\begin{aligned}
Q_{o, k+1} & =Q_{o, k}+\epsilon_{q, k} \\
\rho_{o, k+1} & =\rho_{o, k}+\epsilon_{\rho, k}
\end{aligned}
$$

Note that in this way, the use of the uncertain overflow model (4)-(5) and the settling and scouring functions (7)(8) is circumvented. Furthermore, the noise does not necessarily have to be additive and Gaussian, as the particle filter assumes the most general, nonlinear state-space model $x_{k+1}=f\left(x_{k}, u_{k}, \epsilon_{x}\right), y_{k}=h\left(x_{k}, \epsilon_{y_{k}}\right)$. With the following definition of the augmented state, input and output vectors:

$$
x=\left(\begin{array}{c}
V_{t} \\
m_{t} \\
Q_{o} \\
\rho_{o}
\end{array}\right), \quad u=\left(\begin{array}{c}
Q_{i} \\
\rho_{i}
\end{array}\right), \quad y=\left(\begin{array}{c}
V_{t} \\
m_{t}
\end{array}\right)
$$

the complete nonlinear state-space model becomes:

$$
\begin{aligned}
x_{1, k+1} & =x_{1, k}+T_{s}\left(u_{1, k}-x_{3, k}\right)+\epsilon_{x 1, k} \\
x_{2, k+1} & =x_{2, k}+T_{s}\left(u_{1, k} u_{2, k}-x_{3, k} x_{4, k}\right)+\epsilon_{x 2, k} \\
x_{3, k+1} & =x_{3, k}+\epsilon_{x 3, k} \\
x_{4, k+1} & =x_{4, k}+\epsilon_{x 4, k} \\
y_{k} & =\left(\begin{array}{c}
x_{1, k} \\
x_{2, k}
\end{array}\right)+\left(\begin{array}{c}
\epsilon_{y 1, k} \\
\epsilon_{y 2, k}
\end{array}\right)
\end{aligned}
$$

The objective is to estimate $Q_{o}$ and $\rho_{o}$ on-line.

\section{PARTICle Filter}

The particle filter (PF) uses the model (13) which specifies the probability density functions (PDF) for the state transition function and the measurement function, respectively:

$$
p\left(x_{k} \mid x_{k-1}\right), \quad p\left(y_{k} \mid x_{k}\right) .
$$

The objective is to recursively construct the posterior PDF $p\left(x_{k} \mid y_{k}\right)$ of the state, given the measured output $y_{k}$ and assuming conditional independence of the measurement sequence, given the states. The PF works in two stages:

1) The prediction stage uses the state-transition model in (13) to predict the state PDF one step ahead. The PDF obtained is called the prior.

2) The update stage uses the latest measurement to correct the prior via the Bayes rule. The PDF obtained after the update is called the posterior PDF.
Particle filters represent the PDF by $N$ random samples (particles) $x_{k}^{i}$ with their associated weights $w_{k}^{i}$, normalized so that $\sum_{i=1}^{N} w_{k}^{i}=1$. At time instant $k$, the prior PDF $p\left(x_{k-1} \mid y_{k-1}\right)$ is represented by $N$ samples $x_{k-1}^{i}$ and the corresponding weights $w_{k-1}^{i}$. To approximate the posterior $p\left(x_{k} \mid y_{k}\right)$, new samples $x_{k}^{i}$ and weights $w_{k}^{i}$ are generated. Samples $x_{k}^{i}$ are drawn from a (chosen) importance density function $q\left(x_{k}^{i} \mid x_{k-1}^{i}, y_{k}\right)$, and the weights are updated, using the current measurement $y_{k}$

$$
\tilde{w}_{k}^{i}=w_{k-1}^{i} \frac{p\left(y_{k} \mid x_{k}^{i}\right) p\left(x_{k}^{i} \mid x_{k-1}^{i}\right)}{q\left(x_{k}^{i} \mid x_{k-1}^{i}, y_{k}\right)}
$$

and normalized

$$
w_{k}^{i}=\frac{\tilde{w}_{k}^{i}}{\sum_{j=1}^{N} \tilde{w}_{k}^{j}} .
$$

The posterior PDF is represented by the set of weighted samples, conventionally denoted by:

$$
p\left(x_{k} \mid y_{k}\right) \approx \sum_{i=1}^{N} w_{k}^{i} \delta\left(x_{k}-x_{k}^{i}\right) .
$$

Here, we choose the importance density $q\left(x_{k} \mid x_{k-1}, y_{k}\right)$ equal to the state-transition PDF $p\left(x_{k} \mid x_{k-1}\right)$. The weight update equation (14) then becomes:

$$
\tilde{w}_{k}^{i}=w_{k-1}^{i} p\left(y_{k} \mid x_{k}^{i}\right) .
$$

The PF algorithm is summarized in Algorithm 1.

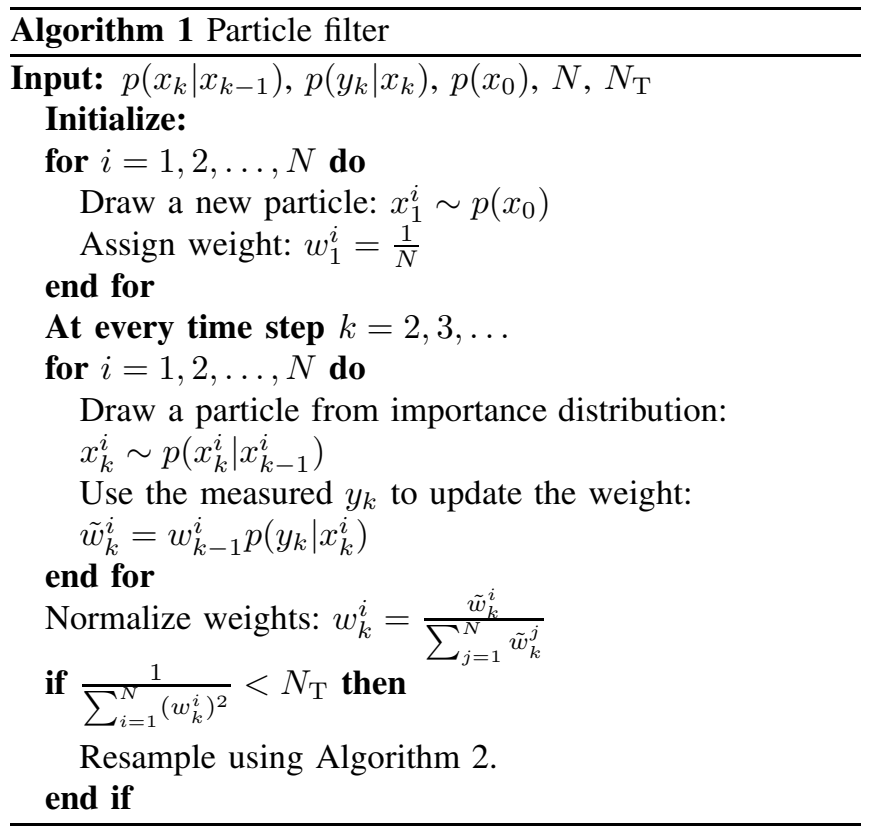

A common problem of PF is the particle degeneracy: after several iterations, all but one particle will have negligible weights. Therefore, particles must be resampled. A standard measure of the degeneracy is the effective sample size:

$$
N_{\mathrm{eff}}=\frac{1}{\sum_{i=1}^{N}\left(w_{k}^{i}\right)^{2}}
$$


If $N_{\text {eff }}$ drops below a specified threshold $N_{\mathrm{T}} \in[1, N]$, particles are resampled by using Algorithm 2.

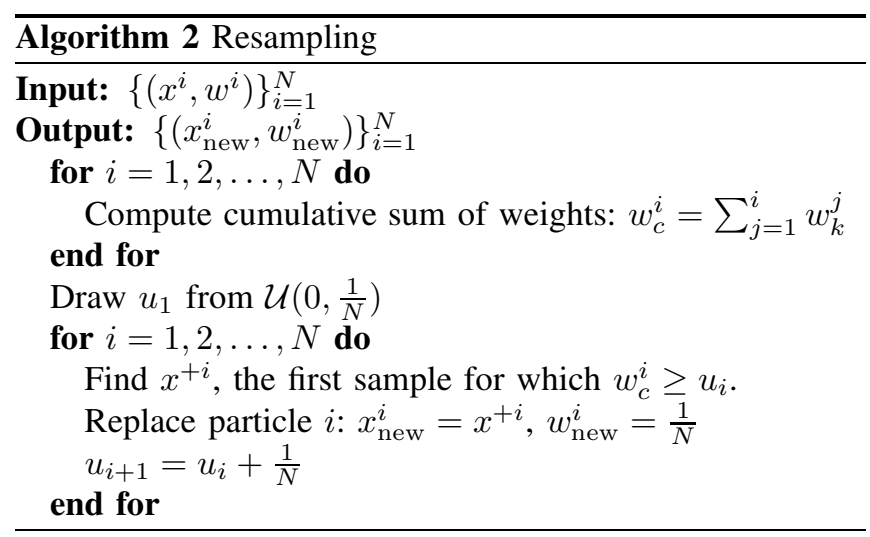

The state estimate is computed as the weighted mean of the particles:

$$
\hat{x}_{k}=\sum_{i=1}^{N} w_{k}^{i} x_{k}^{i} .
$$

For more details on particle filters, refer to [8], [9], [10].

\section{RESULTS}

The PF is applied to both simulated data, obtained with the dynamic sedimentation model described in Section II, and real data recorded during a dredging project. One dredging cycle is selected to illustrate the results. The flow-rate and density of the incoming mixture are shown Fig. 3.
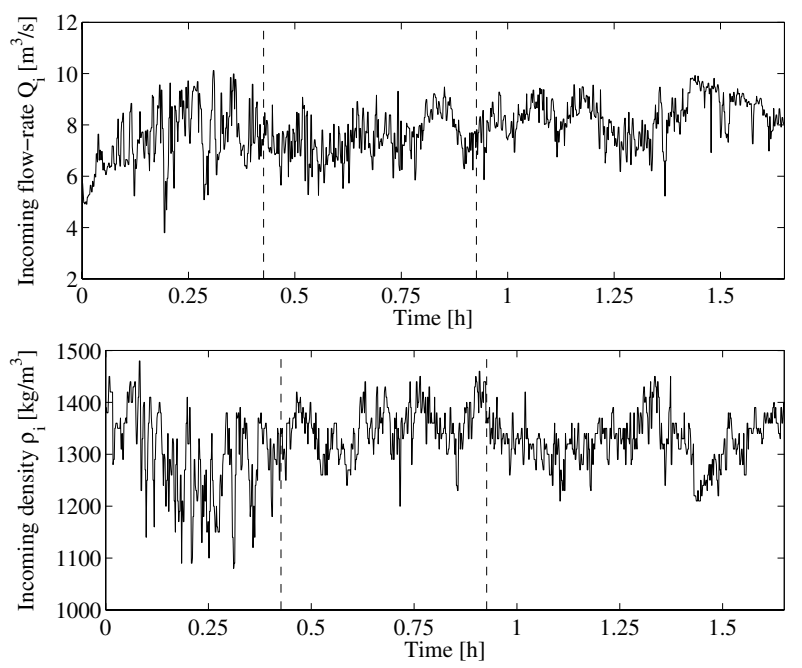

Fig. 3. Flow-rate and density of the incoming mixture.

The loading of the hopper is illustrated in Fig. 4, which shows the time evolution of the measured hopper mass and volume as a consequence of the manipulated overflow height. In the first phase, the total level $h_{t}$ is lower than the overflow level $h_{o}$ and the hopper is filled without any overflow losses (both $V_{t}$ and $m_{t}$ are the result of pure integration). In the second phase, $h_{t}$ becomes greater than $h_{o}$; the steady-state overflow $Q_{o}$ equals the inflow $Q_{i}$ and the total volume $V_{t}$ remains constant. As the overflow density $\rho_{o}$ is lower than the inflow density $\rho_{i}$, the total mass $m_{t}$ still increases. In the third phase, $m_{t}$ reaches the maximum allowable value (given by the maximum laden draught of the ship) and is automatically kept constant by a PID controller which controls the overflow $h_{o}$. In this phase, the volume is decreasing until the hopper is completely filled with sand or the overflow losses become so high that it is no longer economical to continue dredging.
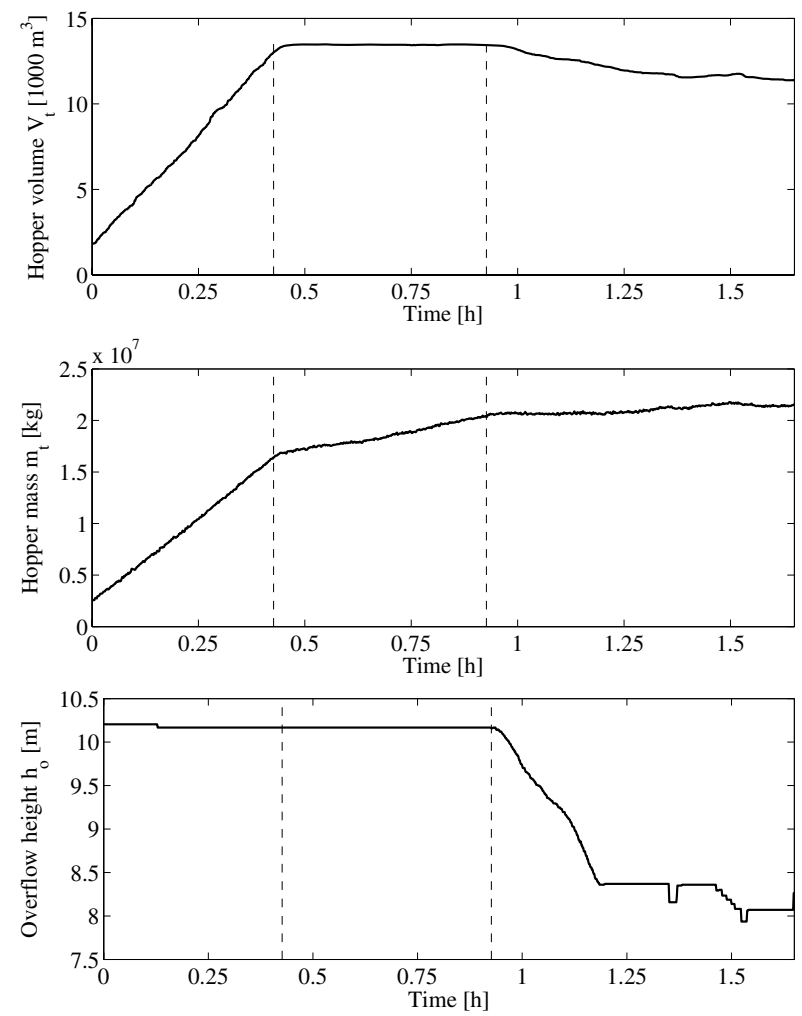

Fig. 4. An example of dredger loading: the evolution of the hopper mass and volume as a consequence of the manipulated overflow height. The vertical dashed lines mark the transitions between the phases.

\section{A. Performance Evaluation}

For the simulated data, the performance of the PF can easily be evaluated by comparing the estimated flow rate and density to the simulated ones. The difference of these two variables is referred to as the estimation error or residual.

However, for the real data, the overflow rate and density measurements are not available (for reasons explained in Section I). We use a rough estimate obtained by off-line computation on the basis of the discretized balance equations (9) and (10). From the volume balance (9), the overflow rate can be estimated by direct computation:

$$
\bar{Q}_{o, k}=Q_{i, k}-\frac{1}{T_{s}}\left(V_{t, k+1}-V_{t, k}\right) .
$$

From the mass balance (10), the overflow density is computed by:

$$
\bar{\rho}_{o, k}=\frac{Q_{i, k} \rho_{i, k}-\frac{1}{T_{s}}\left(m_{t, k+1}-m_{t, k}\right)}{\bar{Q}_{o, k}} .
$$


As the volume and mass measurements are very noisy, an anti-causal first-order low-pass filter is first applied to these signals. The cut-off frequency of the filter was experimentally chosen at $0.001 \mathrm{~Hz}$. The direct computation results shown in Section V-C are all obtained with the filtered data.

\section{B. Simulation}

In order to get some insight in the achievable performance, the PF is first applied to simulated data, obtained with the model of Section II. In Figure 5, the simulated and estimated quantities are compared. Note that only the third phase of the dredging cycle is shown (in the first phase, there is no overflow and in the second phase nearly only water is being discharged overboard).

These results were obtained with $N=1000$ particles and zero-mean Gaussian noise distributions with the following standard deviations: $\sigma\left(\epsilon_{x}\right)=\left[\begin{array}{llll}0, & 3000, & 0.2, & 5\end{array}\right]^{T}$ and $\sigma\left(\epsilon_{y}\right)=[10,12000]^{T}$. These standard deviations were tuned experimentally. Note that there is no need for additional state-transition noise in the volume equation (9), as noise is already added to the flow $Q_{o}$ in the random-walk model (11). The threshold $N_{T}$ for effective sample size is set to 900 (i.e., $90 \%$ of the number of particles $N$ ).
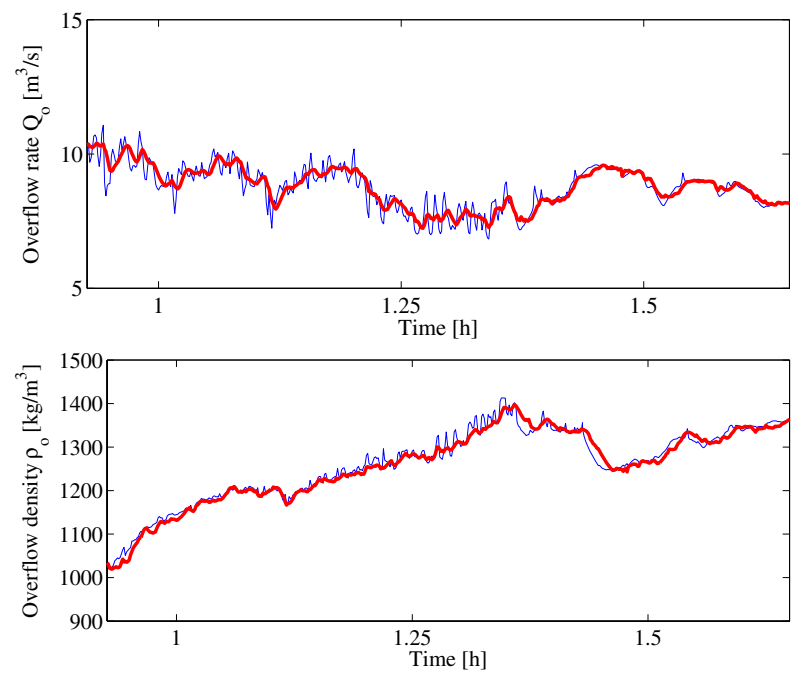

Fig. 5. Simulation results (thin line: simulated variables, thick line: variables estimated by the particle filter).

From Fig. 5, one can already see that the estimation results are very good. This is confirmed by analyzing the distribution of the residuals (Fig. 6). The overflow residual has a mean of $-0.023 \mathrm{~m}^{3} / \mathrm{s}$ and standard deviation of $0.345 \mathrm{~m}^{3} / \mathrm{s}$. The largest estimation errors are about $1 \mathrm{~m}^{3} / \mathrm{s}$ which is about $10 \%$ of the nominal overflow range.

The estimate of the overflow density is slightly biased; the residual has a mean of $3.613 \mathrm{~kg} / \mathrm{m}^{3}$ and standard deviation of $13.226 \mathrm{~kg} / \mathrm{m}^{3}$. The largest estimation errors are about $40 \mathrm{~kg} / \mathrm{m}^{3}$ which is about $10 \%$ of the nominal overflow density range.
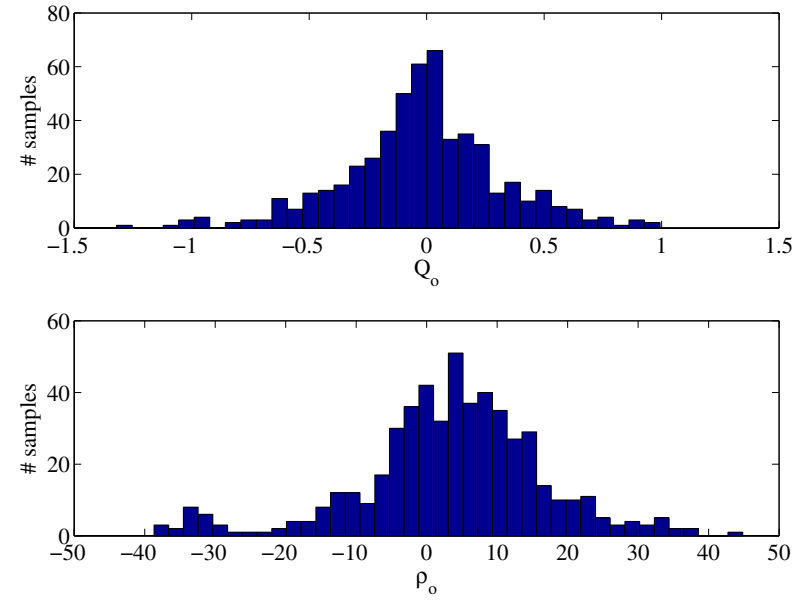

Fig. 6. Simulation results: distribution of the residuals.

\section{Real Data}

Figure 7 shows the $Q_{o}$ and $\rho_{o}$ estimated by the PF in a comparison to the signals obtained by (15)-(16). Note in the first place that the measured flow and density patterns are different from the simulated ones (shown in Fig. 5). This is due to the mismatch between the real process and the mechanistic model presented in Section II.

In Fig. 7, it can further be seen that the PF provides much smoother estimates than the direct computation method. Moreover, the PF can be readily applied on-line. However, one must be aware of the fact that the PF results are influenced by the random sampling of the particles. This influence decreases when more particles are used, but, at the same time, the computational costs become larger. In our application, this is not a problem, as the dredging process is relatively slow.
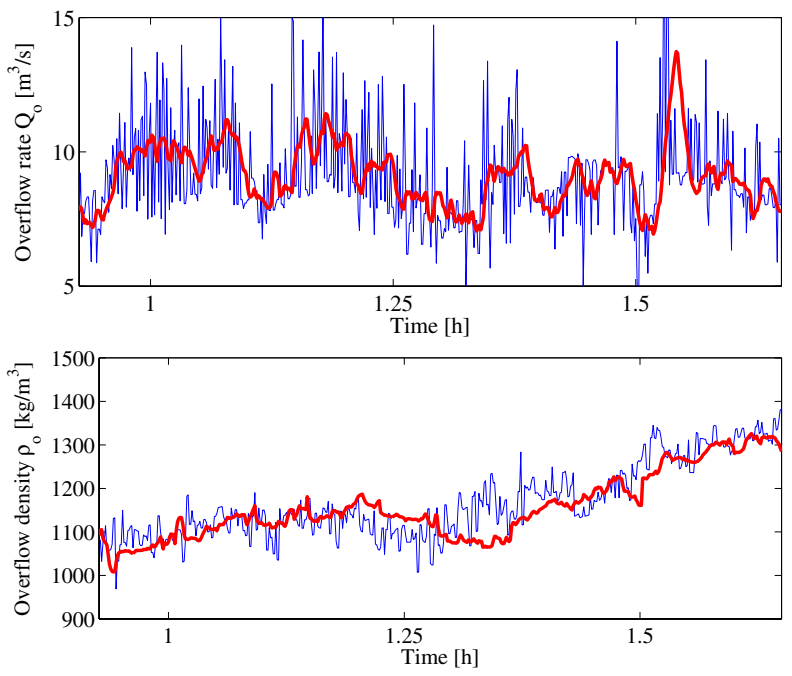

Fig. 7. Results obtained with the measured data (thin line: estimates through the direct computation (15)-(16), thick line: estimates by the particle filter).

Figure 8 gives an impression of the variation in the estimated density trajectories. The gray band delimits the 
minimal and maximal density estimates over 25 independent filter runs on the same data. The bold line is mean of these runs. The PF parameters were the same as with the simulated data.

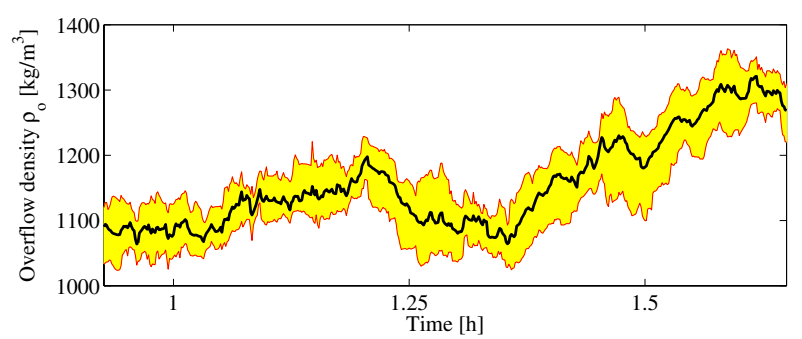

Fig. 8. Variance in the trajectories estimated by the particle filter.

\section{CONCLUSIONS}

A particle filter was applied to the estimation of overflow losses in a hopper dredger. The overflow losses, represented by the overflow mixture density and flow-rate are estimated on the basis of the measured total hopper volume, hopper mass, incoming mixture density and flow-rate.

The proposed approach uses straightforward nonlinear mass balance equations and does not rely on the complex overflow and sedimentation models (4)-(8) which contain many uncertain parameters and functional relationships.

The performance was evaluated in simulations and with real measurements. The results are encouraging, although the random nature of the particle filter results in a relatively large variance in the estimated state trajectories. In our future research, the filter will be used to improve parameter estimation in complex mechanistic models of the hopper sedimentation process. It will also be integrated in a decisionsupport tool for the future use on board of the hopper dredger.

\section{ACKNOWLEDGEMENT}

This research is in part sponsored by Senter, Ministry of Economic Affairs of the Netherlands within the project Artificial Intelligence for the Control of a Hopper Dredger (grant no. TSMA 2017). Zsofia Lendek and Robert Babuška also gratefully acknowledge the support of the BSIK-ICIS project Interactive Collaborative Information Systems, funded by the Dutch Ministry of Economic Affairs (grant no. BSIK03024). The authors also thank the dredging company DEME for supplying the data.

\section{APPENDIX}

This appendix gives the list of symbols used throughout the paper. Subscript $k$ denotes the discrete time index, a hat $\left.{ }^{\wedge}{ }^{\wedge}\right)$ denotes variables estimated by the particle filter and a bar $\left(^{-}\right)$denotes estimates obtained by direct computation.

\section{Variables:}
$h_{m} \quad$ height of the mixture layer
$h_{o} \quad$ height of the overflow pipe
$h_{s} \quad$ height of the sand bed
$h_{t} \quad$ total height of hopper content

$\begin{array}{ll}m_{s} & \text { mass of sand bed } \\ m_{t} & \text { total hopper mass } \\ Q_{i} & \text { incoming mixture flow-rate } \\ Q_{o} & \text { outgoing mixture flow-rate } \\ u & \text { input variable } \\ V_{t} & \text { total hopper volume } \\ x & \text { state variable } \\ y & \text { output variable } \\ \epsilon & \text { noise disturbance } \\ \rho_{i} & \text { incoming mixture density } \\ \rho_{m} & \text { density of mixture in hopper } \\ \rho_{o} & \text { outgoing mixture density }\end{array}$

\section{Parameters:}

$\begin{array}{ll}A & \text { hopper area } \\ g & \text { acceleration due to gravity } \\ k_{c} & \text { scouring parameter } \\ k_{o} & \text { parameter for free overflow } \\ k_{o}^{\prime} & \text { full overflow parameter } \\ N & \text { number of particles } \\ N_{\mathrm{T}} & \text { resampling threshold } \\ v_{s} & \text { undisturbed settling velocity } \\ T_{s} & \text { sampling period } \\ \beta & \text { exponent in } f_{s} \\ \rho_{q} & \text { quartz density } \\ \rho_{s} & \text { density of sand bed } \\ \rho_{w} & \text { water density }\end{array}$

\section{Probabilities and distributions:}

$p(\cdot) \quad$ probability or PDF

$p(\cdot \mid \cdot) \quad$ conditional probability or PDF

$q(\cdot \mid \cdot) \quad$ proposal (importance) density function

$\mathcal{U}\left(u_{1}, u_{2}\right)$ uniform distribution

$\mathcal{N}(m, \sigma)$ normal distribution

$\sigma(\cdot) \quad$ standard deviation of a random variable

\section{REFERENCES}

[1] T. Camp, "Sedimentation and the design of settling tanks," in Trans. ASCE, 1946, pp. 895-936.

[2] T. Yagi, "Sedimentation effects of soil in hopper," in Proceedings of WODCON world dredging conference, 1970.

[3] S. Ooijens, "Adding dynamics to the camp model for the calculation of overflow losses," Terra et Aqua, no. 76, pp. 12-21, September 1999.

[4] C. van Rhee, "On the sedimentation process in a suction hopper dredger," Ph.D. dissertation, TU Delft, 2002.

[5] J. Franzini, Fluid Mechanics with Engineering Applications. McGraw-Hill, 1997.

[6] J. F. Richardson and W. N. Zaki, "Sedimentation and fluidisation: Part I," Transactions of the Institution of Chemical Engineers, vol. 32, pp. 35-53, 1954.

[7] S. Ooijens, A. de Gruijter, A. Nieuwenhuijzen, and S. Vandycke, "Research on hopper settlement using large-scale modelling," in Proceedings of the CEDA Dredging days 2001, 2001, pp. 1-11.

[8] A. Doucet, N. de Freitas, and N. Gordon, Sequential Monte Carlo Methods in Practice. Springer Verlag, 2001.

[9] S. Arulampalam, S. Maskell, N. Gordon, and T. Clapp, "A tutorial on particle filters for on-line nonlinear/non-gaussian bayesian tracking," IEEE Transactions of Signal Processing, vol. 50, no. 2, pp. 174-188, 2002

[10] B. Ristic, S. Arulampalam, and N. Gordon, Beyond the Kalman Filter. Particle Filters for Tracking Applications. Artech House, Boston, 2004. 